\title{
PSR B0656+14: Combined Optical, X-ray \& EUV Studies
}

\author{
Aaron Golden, Andy Shearer \\ The National University of Ireland, Galway, Newcastle Road, Galway, \\ Ireland
}

Jerry Edelstein

Space Sciences Laboratory, UC Berkeley, USA

\begin{abstract}
.
PSR B0656+14's high energy emission is consistent with that of combined magnetospheric and thermal (surface \& polar cap) emission. Uncertainties with the radio-derived distance and $\mathrm{X}$-ray instrumentation sensitivities complicate a definitive thermal characterisation however. A re-analysis of combined ROSAT/EUVE archival data in conjunction with integrated \& phase-resolved optical photometry is shown to constrain this characterisation.
\end{abstract}

\section{Introduction}

Considerable uncertainty remains regarding the fundamental thermal parameters $\left(T, N_{H} \& R / d\right)$ for PSR B0656+14. Radio derived DM estimates (790土 190 pc) disagree with the best $N_{H}$ model fits $(250-550 \mathrm{pc})$. Reported calibration uncertainties associated with the low energy channels of the ROSAT PSPC compromise the latter - although agreement between other ROSAT PSPC \& observed EUVE fluxes obtained via a correction (e.g. for RX J185635-3754, Walter \& An, 1998). We outline the results of such a correction to the existing PSPC datasets archived for PSR B0656+14 via substitution of the low energy channels with measured $E U V E$ fluxes, and by incorporating independently derived constraints to the Rayleigh-Jeans tail in the optical, discuss the implications for the neutron star's thermal parameters.

\section{Technical \& Analytical Overview}

Optimum thermal fits for $T_{\text {soft }}, T_{\text {hard }}, N_{H}, R / d$ were obtained for the archived ROSAT PSPC data alone and the PSPC data with the suspect low energy channels substituted with the archival normalised EUVE flux. This substitution results in a significant change in solution space, as shown in Figure 1 (Edelstein et al. 1999). Based on integrated optical photometry, Pavlov et al. (1997) fitted a two component nonthermal/thermal model, the thermal fit defined by a parameter $G \equiv T_{10^{6} \mathrm{~K}}\left(R_{10 \mathrm{~km}} / d_{500 \mathrm{pc}}\right)^{2}$ where $G=[1-7]$ (see Figure 1). A $1 \sigma$ upper limit on the unpulsed component from the optical $B$ band light curve 

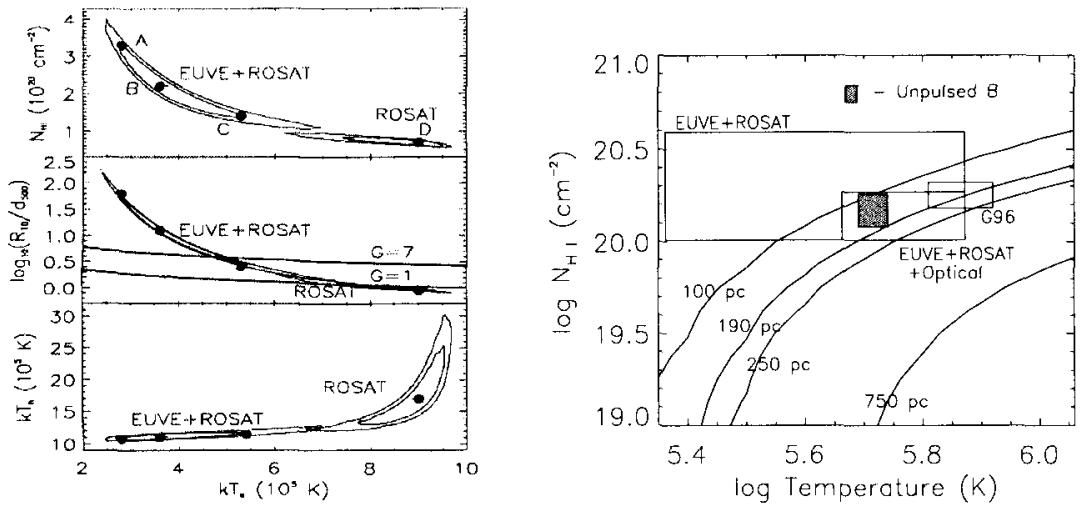

Figure 1. Left: Model Fits to $T_{h}, N_{H}, R / d$ vs. $T_{s}$ at the $90 \%$ and $99 \%$ confidence interval for ROSAT and EUVE+ROSAT datasets. The range of best-fit $G$ parameters are indicated. Right: $\left(N_{H}, T_{s}\right)$ space constrained by EUVE \& ROSAT, and incorporating the optical constraints to $G$. Curves are locii of constant $\left(N_{H}, T_{s}\right)$ based on the observed EUVE count rate. Previous solution of Greiveldinger et al. (1986) is marked.

of Shearer et al. (1997) limits $G \leq 4.4,4.8$ and 5.2, based on various optical extinction models to the pulsar (Golden, 1999). These optical results yields tighter constraints on parameter space, as can be seen.

\section{Discussion \& Conclusions}

Combining the EUVE \& ROSAT datasets in this way yields new solutions in parameter space that are further constrained independently via recent optical work. Assuming a simple blackbody form then $T_{\text {surface }} \geq 5.0 \times 10^{5} \mathrm{~K}$ and for the $N_{H}$-derived distances of $[250-280] \mathrm{pc}, R_{\infty} \leq[17.7-14.7] \mathrm{km}$. Using the estimate of $R_{\infty} \sim 9.5_{-2.0}^{+3.5} \mathrm{~km}$ for Geminga as a working upper limit (Golden \& Shearer, 1999) places PSR B0656+14 at a distance of no less than $d=152_{-32}^{+55}$. This suggests the possibility of parallax observations to independently derive $d$, with immediate implications for the $R$ parameter, and consequently models of the condensed matter equation of state.

\section{References}

Edelstein, J., Seon, K.-I., Golden, A., \& Kwok-in, K., 1999, sub. ApJ Golden, A., 1999, Ph.D. Thesis, National University of Ireland, Galway Golden, A., \& Shearer, A., 1999, A\&A, 342, L5

Greiveldinger, C., Camerini, U., Fry, U., et al., 1986, ApJ, 465, 35

Pavlov, G.G., Welty, A.D., \& Cordova, F.A. 1997, ApJ, 489, L75

Shearer, A., Redfern, R.M., et al. 1997, ApJ, 487, L181

Walter, F.M., \& An, P. 1998, AAS, 192, 82.07 